\section{Psoriasis - Politik - Kunst - Mode - Krankheitsbürde - Lebensqualität}

oder: Was uns die Schuppenflechte Prominenter lehrt
H. Meffert

E. Rowe

\section{Psoriasis - Politics - Arts - Fashion - Burden of Disease - Quality of Life \\ Or: What we are Taught by the Psoriasis of Famous People}

\section{Zusammenfassung}

Besprochen wird die Psoriasis einiger Prominenter - der Einsiedler Elias, der Feldhauptmann Naaman, J. Updike, V. Nabokov, J.-P. Marat, J. W. Stalin, Z. Leander, K. Holstein, L. Le Floch-Progent und andere. Erstaunlicherweise fällt es auch ausgesprochen intelligenten, zielstrebigen und wohlhabenden Psoriasiskranken oft schwer, essenzielle Kenntnisse über den Umgang mit der Krankheit zu erlangen. Auch betroffene Prominente leiden wegen ihrer unzureichenden Kenntnisse und unzulänglicher Therapie oft unnötig. Der informierte Psoriatiker kennt seine Krankheit. Er kann mit ihr und ihrer Behandlung gut umgehen und kämpft erfolgreich für seinen beruflichen und sozialen Status. Therapie und Therapeut werden vom Betroffenen gelegentlich ganz anders reflektiert, als das der behandelnde Arzt annimmt. Das sollte uns nachdenklich stimmen und Anlass für Änderungen sein.

\section{Einleitung}

Winston Churchill (1874-1965), der Einsiedler Elias (um 1230 bei Eisenach), Art Garfunkel (geb. 1941), Karin Holstein (Geburtsjahr unbekannt), Zarah Leander (1907-1981), Jean Paul Marat (1743-1793), Vladimir Nabokov (1899-1977), der Feldhauptmann Naaman (Altes Testament, 2 Könige 5,1-14), Dennis Potter (1934-1995), Romy Schneider (1938-1982), Jossif Wissarionowitsch Stalin (1879-1953), August Strindberg (1840-1912), John Updike (geb. 1932) und viele weitere Berühmtheiten sollen an Psoriasis gelitten haben. Die Schuppenflechte ist eine weit verbreitete Krankheit. Deshalb ist es keineswegs verwunderlich, wenn auch besonders Schöne, Reiche, Kluge, Berühmte, Boshafte oder Einflussreiche von ihr nicht verschont bleiben.

\section{Abstract}

Discussion of the psoriasis of some famous people - the hernit Elias, Captain Naaman, J. Updike, V. Nabokov, J.-P. Marat, J. V. Stalin, Z. Leander, K. Holstein, L. Le Floch-Progent and others. Surprisingly, even intelligent, determined and wealthy psoriatics often have difficulties in getting essential knowledge on the handling of the disease. Also some famous persons suffer needlessly because of their insufficient knowledge and inadequate therapy. Informed psoriatics know their illness. They know how to deal with it and its treatment. And they struggle successfully for their professional and social state. Therapy and therapeut are sometimes reflected by the people concerned in quite a different way of that which the therapeut assumes. This should make us think and be reason for modification.
Doch was kümmert den gewöhnlichen Sterblichen - ob an Psoriasis erkrankt oder nicht - die Schuppenflechte Prominenter? Er sollte sich dafür interessieren. Dann wird er staunend erkennen, dass man es trotz und vielleicht auch gerade wegen Psoriasis beispielsweise bis zum Idol bringen kann. Auf welche Art und Weise kommt eine solche unerwartete Karriere zustande? Glücklicherweise sind nicht wenige Prominente extrovertiert. Frank und frei plaudern sie über ihre Erfahrungen mit der Krankheit. Andere sind zudem noch sensibel. Von ihnen kann man Unerwartetes, gar Erstaunliches über Schaden und Nutzen (!) der Krankheit hören. Das geht so weit, dass einige davon überzeugt sind, es ohne Psoriasis nie so weit gebracht zu haben.

Korrespondenzadresse

Prof. Dr. med. H. Meffert - c/o Hautarztpraxis Dr. med. E. Rowe \& Dr. med. I. Vinogradova .

Potsdamer Chaussee 80, 14129 Berlin-Nikolassee·E-mail: hans.meffert@web.de

Bibliografie

Akt Dermatol 2004; 30: 504-509 @ Georg Thieme Verlag KG Stuttgart • New York ·

DOI 10.1055/s-2004-826044 · ISSN 0340-2541 
Niemand kann den Eindruck, den der Arzt und seine Mitarbeiter auf den Betroffenen machen, so gut formulieren wie ein wortgewaltiger Künstler. Nebenbei bemerkt ist der Zorn der freien Rede zudem für den Redner heilsam und deshalb in solchen Fällen erwünscht. Kommt dann noch ein bei Prominenten gar nicht so seltener Mangel an Zurückhaltung hinzu, so können gerade wir Hautärzte aus derartigen Schilderungen erfahren, wie wir gar nicht so selten gesehen werden. Das wiederum ist lehrreich und nützlich.

\section{Jahrtausendelang verkannt und verwechselt}

Im Altgriechischen bedeutet Psora soviel wie Jucken, Krätze oder auch Räude. Die Bezeichnung wurde zeitweilig für Hautkrankheiten wie Skabies, Impetigo, Tinea oder Lepra verwandt. Noch heute kommt es vor, dass Psoriasis mit Lepra verwechselt wird. Dieser Irrtum hat Lebensläufe dramatisch verändert. Erst Robert Willan (1757-1812) und Ferdinand von Hebra (1816-1880) grenzten die Psoriasis vulgaris von der Lepra ab und definierten sie im heutigen Sinne. Willan bezeichnete einzelne, großflächige Herde noch als Lepra Graecorum, konfluierende Herde als Psora leprosa [1].

Leitlinien der Lepra-Diagnostik und die Beschreibung sozialer Konsequenzen der Erkrankung finden sich bereits im Alten Testament. Entsteht aber auf der Glatze des Hinterkopfes oder über der Stirn ein hellroter Fleck, so ist es Aussatz... Der Priester muss ihn für unrein erklären; er ist an seinem Kopf von Aussatz befallen. Der Aussätzige ... soll eingerissene Kleider tragen und das Kopfhaar ungepflegt lassen; er soll den Schnurrbart verhüllen und ausrufen: Unrein! Unrein! ... Er soll abgesondert wohnen, außerhalb des Lagers soll er sich aufhalten [2].

Es muss auch angenommen werden, dass nicht wenige als aussätzig fehldiagnostizierte Psoriatiker in der Notgemeinschaft der Leprakranken tatsächlich an Lepra erkrankten und daran verstarben.

Ein Wunder trug sich zu bei einem prominenten Kranken. Naaman, der Feldherr des Königs von Aram, ... war tapfer aber an Aussatz erkrankt. Elischa ... ließ ihm sagen: Geh und wasche dich siebenmal im Jordan! Dann wird dein Leib wieder gesund, du wirst rein ... So ging er also zum Jordan hinab und tauchte siebenmal unter... Da wurde sein Leib gesund wie der Leib eines Kindes und er war rein [3]. Das siebenmalige Bad im Jordan war eine kurmäßige Anwendung, die mit aller Wahrscheinlichkeit tagsüber am sonnigen Ufer des Flusses stattfand. Vielleicht handelt es sich hier sogar um die Erstbeschreibung der Balneophototherapie der Psoriasis. Denn damals war Lepra eine progrediente, unheilbare Krankheit. Der Feldhauptmann Naaman aber genas. Das hatte er mit dem Einsiedler Elias, genannt der arme Eli, gemein [4]. Dessen wundersame Heilung vom angeblichen Aussatz wurde allerdings nicht der Heilkraft eines besonderen Bades zugeschrieben, sondern aufopfernder Pflege und Salbenbehandlung, die ihn bis ins Bett der Elisabeth von Thüringen gelangen ließ (Abb.1).

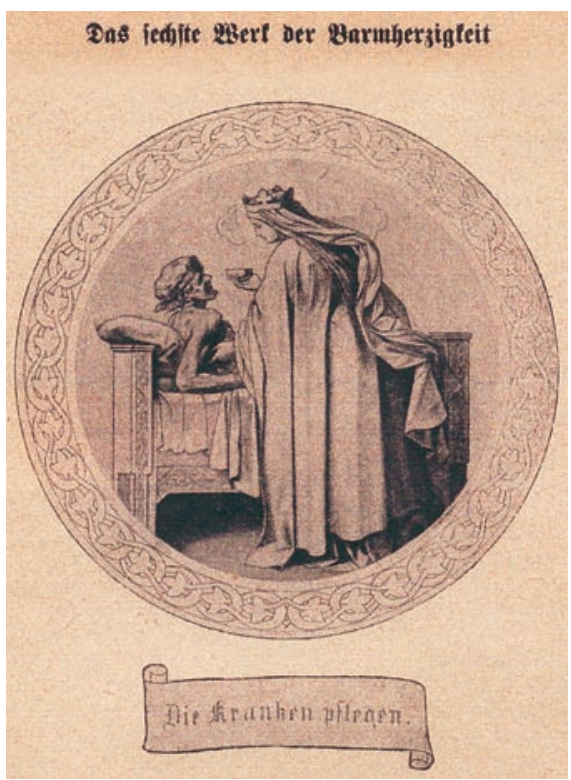

Abb. 1 Die sieben Werke der Barmherzigkeit der Heiligen Elisabeth. Wandgemälde auf der Wartburg von Moritz von Schwind. In: Kunstgaben für Schule und Haus. Herausgegeben von W. Günther (Hamburg), Heft 14. Verlag von Georg Wigand, Leipzig 1911.

\section{Wie ein Blitz aus heiterem Himmel}

In der Regel bricht die Schuppenflechte erstmals im frühen Erwachsenenalter aus. Dann schlägt sie ein wie ein Blitz aus heiterem Himmel. Dem Betroffenen wird klar, dass ab sofort ein wesentlicher Part in seinem Leben von einer unerwarteten Größe übernommen wurde - von seiner Psoriasis. Die Auseinandersetzung mit der Krankheit beginnt oft heftig, gefolgt von depressiver Verstimmung, begleitet von immer wieder aufkeimender Hoffnung und zeitweiliger Resignation. Die Reaktion der Umgebung wie auch therapeutische Erfolge und Misserfolge modifizieren den langwierigen Vorgang, der als Krankheitsverarbeitung bezeichnet wird. Die Mehrzahl der behandelnden Ärzte geht davon aus, dass aktive Auseinandersetzung besonders günstig ist. Der Erkrankte soll zum gelernten Psoriatiker (Otto Braun-Falco) werden, indem er sich umfassend informiert über das Wesen der Erkrankung, über Remission und Rezidiv, Therapie, auslösende Faktoren, über vorteilhaftes Verhalten in Alltag, Beruf, Freizeit und Urlaub. Aus freimütigen Schilderungen Prominenter lässt sich hierzu Wesentliches erfahren. Das betrifft vor allem die Formen der Auseinandersetzung mit der Krankheit und mit den veränderten Lebensbedingungen inklusive Therapie und Therapeut. Es betrifft auch die Frage, ob und wie die Schuppenflechte Einfluss auf den weiteren Lebensweg und wesentliche Aktivitäten und Entscheidungen nehmen kann.

\section{Die Schriftsteller}

Im Kapitel „Aus dem Tagebuch eines Aussätzigen“ schildert John Updike seine Psoriasis [5]. Der Verlauf der Krankheit ist wie folgt: Flecken, Placken und Lawinen überschüssiger Haut, die von der Derma dank eines unbedeutenden aber beharrlichen Fehlers in ihrem metabolischen Code produziert werden, dehnen sich aus und wandern langsam über den Körper wie Flechten auf einem Grabstein. Ich bin silbern, schuppig. Lachen abgeblätterter Schuppen bilden sich, wo immer ich mich zur Ruhe lege. Jeden Morgen sauge ich im Bett Staub. Meine Qual ist hauttief: keine Schmerzen, nicht mal 
ein Jucken. Wir Aussätzigen leben lange und sind ironischerweise in anderer Hinsicht gesund. Im Schub dominiert die Schuppenflechte nahezu alles, so auch die Beschreibung des Hautarztes. Seine eigene Haut trägt die staubig rosigen Reste von Sommersonnenbräune. Sein Kopf ist makellos kahl und traumhaft glatt. Ich frage mich, welche Perversität ihn in die Dermatologie getrieben hat. Die PUVA-Therapie löst ungewöhnliche Empfindungen aus. Die Lichtbox hat sechs Seiten, die mit vertikalen Röhren bestückt sind. ... Ein Röhren wenn es losgeht, so dass man astronautische Anwandlungen hat, ebenso Anwandlungen von Absurdität, ein stehender Nackter wie in einem „,gewagten“ Stück, wo die Bühnenlichter die Zuschauer verschluckt haben. ... Der Tanz ist kurz; die erste Dosis beträgt nur eine Minute. Die Box gibt ein böses, tadelndes Schnurren von sich, wenn sie sich abstellt. Stets und ständig versucht der Betroffene, die kranke Haut zu verbergen. Meine Hände würden mich verraten, aber während des Essens bewege ich sie ständig, um ihr Aussehen zu verwischen. Auch als sich die Haut unter PUVA-Therapie zu bessern beginnt, bleibt die tief sitzende Furcht. Ich schildere meine Empfindung, dass der von meiner Haut verjagte Aussatz in tiefere Gewebe flieht und dort nur darauf wartet, in noch ekelhafterer und teuflischerer Form wiederaufzuerstehen. Gut eine Woche später ist die Haut glatt. Ich bin schön. Ich ziehe mich dauernd wieder aus, um sicher zu sein. Sogar auf den Schienbeinen ist der Aussatz verschwunden, ein feines Krakelee trockener Haut hinterlassend, wie bei Tang-Porzellan, das Badeöl bessern wird.

Während John Updike seine Psoriasis ausführlich beschrieb, widmete ihr Vladimir Nabokov in seinem Roman „Ada“ nur weniger als eine Seite [6]. Dort geben sich zwei Psoriasiskranke Tipps: Quecksilber! Höhensonne wirkt Wunder. Ansonsten werden noch heiße Bäder empfohlen, zweimal monatlich oder des öfteren, und das Meiden von Gewürzen.

Ein Allgemeinmediziner sah die Psoriasis sogar als eine Metapher für den kreativen Prozess an. Sie sei das Ergebnis der Implosion des Künstlers, und die Romane über Psoriasis würden die Idee kultivieren, dass der psoriatische Plaque die Achillesferse des introvertierten Individualisten sei, eines Künstlers, der die Welt vom Elfenbeinturm seiner Psoriasis aus betrachtet [7].

\section{Der Revolutionär}

Der Arzt, Naturforscher, Verleger, Journalist und Berufsrevolutionär Jean-Paul Marat und dessen Hautkrankheit wurden der Öffentlichkeit durch das im Jahre 1964 in Berlin uraufgeführte Drama von Peter Weiss [10] wieder ins Gedächtnis gerufen. Der gebürtige Schweizer Marat hatte in Paris Medizin studiert und war dann zehn Jahre nach England und Wales gegangen, wo er promovierte, zum Modearzt avancierte und auch über Optik und Elektrizität publizierte. 1790 - 1792 verbarg er sich als Herausgeber einer radikal revolutionären Zeitschrift bis zum Sturz der Monarchie auch wörtlich im Untergrund von Paris. In dieser Zeit begannen seine Hautprobleme, bei denen es sich am ehesten um Psoriasis, später Erythrodermia psoriatica, gehandelt hat $[8,9]$. Noch heute scheiden sich die Geister an der Frage ob Marat ein Märtyrer oder Monster war. Seit 1774 verschlechterte sich der Hautzustand rapide. Im Drama von Peter Weiss heißt es dazu:

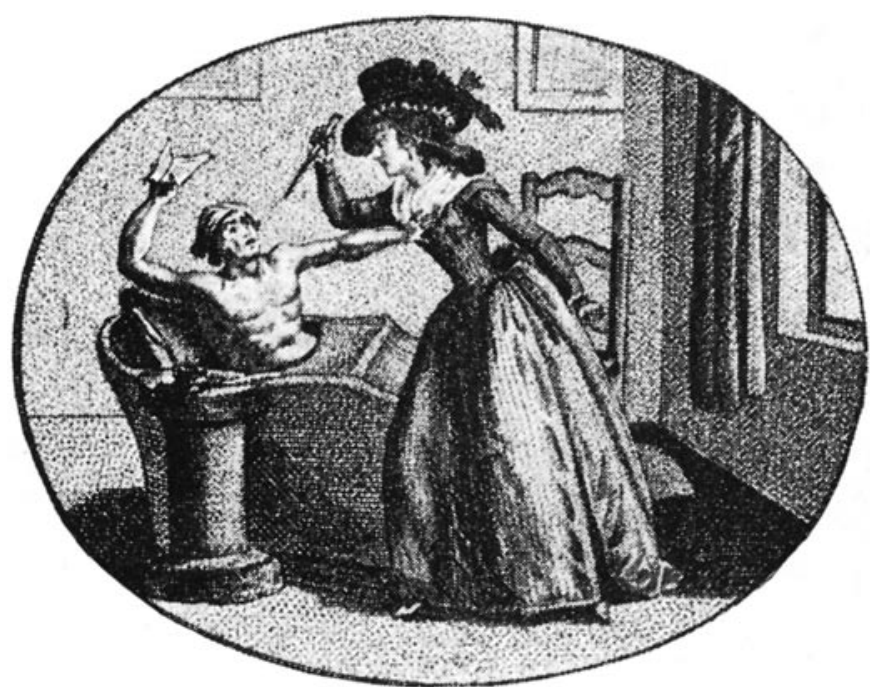

Abb. 2 Die Ermordung des Marat. Illustration aus [10].

Seine Haut ist flammig und gelb weil von einem Ausschlag entstellt

Das kühle Wasser in dem er sitzt lindert das Fieber das ihn erhitzt.

Entzündung und Juckreiz hatten zugenommen, Fieber war hinzugekommen. Um seine brennende Stirn zu kühlen, benutzte er ständig mit Essigwasser getränkte Binden. Die ebenfalls ständig gebrauchten Bäder sorgten für Linderung des Juckreizes und senkten das Fieber. Daher verließ er die von einem Tuch bedeckte Sitzbadewanne nur selten [10]. Es ist nicht bekannt, ob das Badewasser Zusätze enthielt. Marat wurde in seiner Badewanne am 13. Juli 1793 von der Girondistin Charlotte Corday erstochen (Abb. 2).

\section{Der Politiker}

Zeitlebens wurde Jossif Wissarionowitsch Stalin (Abb. 3) von Krankheiten geplagt [11]. Mit sieben Jahren erkrankte er schwer an Windpocken, die im Gesicht viele auffällige Narben hinterließen. Drei Jahre später wurde er von einem Pferdewagen überfahren. Der linke Arm brach mehrfach. Nach einer Osteomyelitis wuchs der Arm verkürzt und verkrümmt zusammen. In den frühen zwanziger Jahren erkrankte Stalin vermutlich an Tuberkulose. 1953 erlitt er einen Schlaganfall, den seine Tochter Swetlana und sein Nachfolger N. S. Chruschtschow dokumentierten. Es wird angenommen, dass Stalins Tod durch Vergiftung herbeigeführt wurde.

Im Jahre 1990 gab der langjährige sowjetische Gesundheitsminister B. Petrowski der Zeitschrift "Ogonjok“ ein Interview, in dem er aussagte, dass Stalin seit früher Jugend an Schuppenflechte litt. Stalins Sekretär und Dolmetscher von 1942 bis 1954, V. Bereskow, hatte „weißliche, hautartige Stücke an den Schultern und verfärbte Flecken an den Händen" beobachtet. Der Dichter Osip Mandelstam schrieb 1934 von den „feisten, wurmartigen Fingern des Kreml-Bewohners“. Stalin reagierte äußerst 


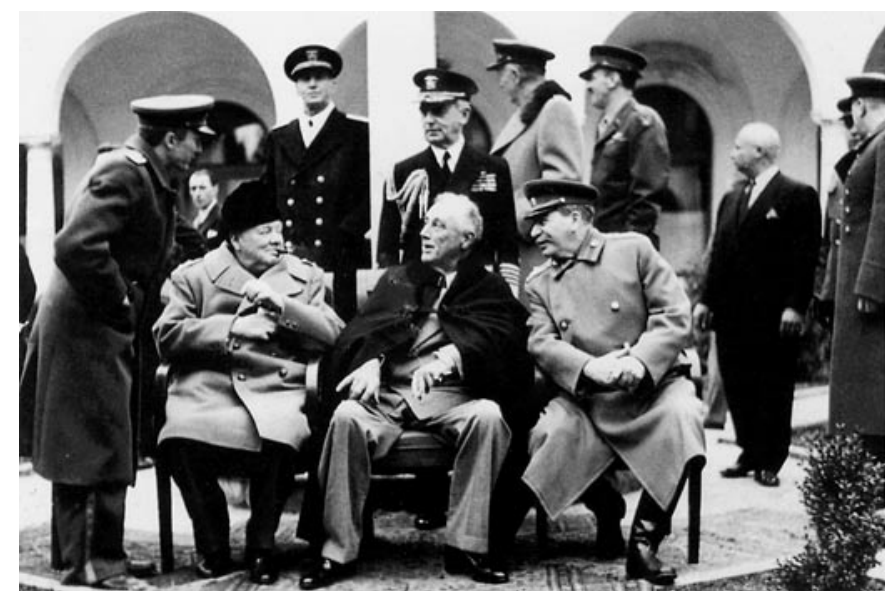

Abb. 3 Stalin, Churchill und Roosevelt. Drei Männer, die die Welt veränderten. Zwei davon hatten Schuppenflechte. In: Adatto M. Lebendige Haut. Schmucktätowierungen und Dermatologie. Editiones Roche, Basel 1993.

wütend und ließ Mandelstam nach Sibirien verbannen, wo dieser verstarb.

In den dreißiger Jahren wurde Stalins Psoriasis erstmals mit „Lysaten“ behandelt. Das sind Produkte aus verschiedenen Organen, die im sauren Milieu unter hohem Druck durch Einwirkung proteolytischer Enzyme entstehen. Es wurde behauptet, dass Lysate die Funktionen ihres Ursprungsorgans spezifisch stimulieren könnten. So sollten z.B. Hühner durch Lysate aus Eierstöcken zum Legen von mehr Eiern veranlasst werden und Kühe durch Lysate aus Brustdrüsen mehr Milch produzieren. Es ist nicht bekannt, ob die therapeutisch eingesetzten Lysate tierischen oder menschlichen Ursprungs waren.

Hergestellt wurden solche Lysate im Institut des Allgemeinarztes I. N. Kasakow. Den Worten eines seiner Mitarbeiter zufolge war dieser „ein Quacksalber, für den es unheilbare Krankheiten nicht gibt.“ Nach der Remission der Stalin'schen Schuppenflechte wurde das Moskauer Staatliche Institut für Haut- und Geschlechtskrankheiten flugs in ein sogenanntes Stoffwechsel-Institut umgewandelt und an Kasakow übergeben. Bald rezidivierte Stalins Schuppenflechte. Neuerliche Behandlungsversuche mit Lysaten schlugen fehl. Kasakow fiel in Ungnade und geriet mit anderen russischen Prominentenärzten in den Strudel der „Großen Prozesse“. Damals stabilisierte Stalin seine Macht, indem er sich echter und vermeintlicher Feinde in Schauprozessen entledigte. Wie die anderen angeklagten Ärzte wurde Kasakow unmittelbar nach dem Schuldspruch „wegen Mordes“ hingerichtet.

Es drängt sich die Frage auf, ob Stalins ungeheuerliche Aktivitäten in ursächlichem Zusammenhang mit seiner Schuppenflechte gesehen werden dürfen. Wohl nicht. Aber das pockennarbige Gesicht und der verunstaltete Arm mögen negative psychische Auswirkungen gehabt haben, die sein paranoisches Verhalten gegenüber den Ärzten und seine Furcht vor der Medizin erklären könnten. Übrigens steht in den Krankenakten auch, dass Stalin 1,62 m groß war und kurze, krumme Beine hatte.

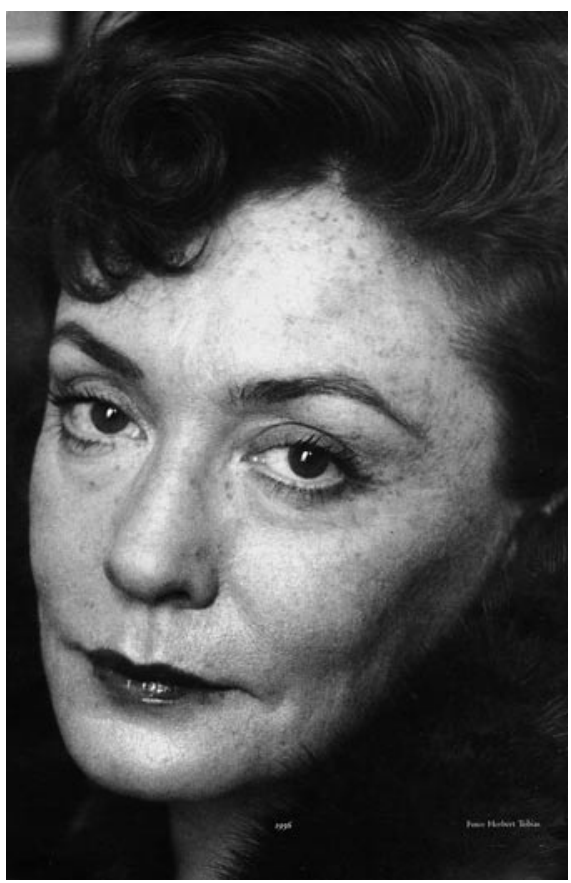

Abb. 4 Zarah Leander im Alter von 49 Jahren. In: Seiler P. Ein Mythos lebt. Zarah Leander. Verlag Druckpunkt, Berlin 1991.

\section{Die Sängerin}

Es gibt Melodien, Ohrwürmer, denen man sich einfach nicht entziehen kann. Auch dann nicht, wenn man mit dem Verständnis des zugehörigen Textes so seine Schwierigkeiten hat. Immer wieder widmen die Medien ihre Aufmerksamkeit Zarah Leander, der Schwedin mit der sonoren, wodka-, später whisky-geschwängerten Stimme. Das liegt wohl einerseits an den nach wie vor verführerischen Melodien, die zumeist von Michael Jary komponiert worden waren und später auch von Udo Lindenberg, Nina Hagen, Erika Pluhar, Milwa, Romy Haag und André Heller gesungen wurden. Andererseits geht es um Vergangenheitsbewältigung. Es hieß, Zarah Leander sei eine Diseuse von Goebbels Gnaden gewesen. Möglicherweise hatte der Reichspropagandaminister die beliebte UFA-Schauspielerin auserkoren, um Hollywood-Stars wie Greta Garbo und Marlene Dietrich vergessen zu machen.

Ich weiß, es wird einmal ein Wunder gescheh'n. Nach der Lektüre des Buchs der Tochter Michael Jarys [12] erscheint es plausibel, dass das erwartete Wunder mit der gleichermaßen unerschöpflichen wie unerfüllten Liebe zu Michael Jary zu tun haben sollte. Davon geht die Welt nicht unter, sieht man sie auch manchmal grau. Diese Zeile aus einem weiteren Erfolgstitel der Leander passt recht gut zu einem Menschen, der sich unverzagt mit einer hartnäckigen Krankheit auseinandersetzt. Vor nahezu 30 Jahren hatte Heinz-Egon Kleine-Natrop, Hautarzt in Dresden, die Schuppenflechte der Leander erwähnt [13]. Auf einem Schwarzweißfoto der 49-jährigen finden sich besonders auf der Stirn zahlreiche, an Sommersprossen erinnernde Flecke (Abb. 4). Einige davon sind deutlich größer, bizarr begrenzt und unterschiedlich pigmentiert. Diese sogenannten PUVA-Sommersprossen sind die Folge übermäßiger UV-Einwirkung, beispielsweise nach jahrelanger UV-Therapie. 
Den Kinogängern wie den Leander-Biographen blieb diese Seite ihres Idols verborgen. Doch in den Memoiren der Diva kommt das Wort „Psoriasis“ vor, wenn auch nur ein einziges Mal: Da leide ich zeitweise an Psoriasis, einer zwar nicht lebensgefährdenden Krankheit, die aber einen ständigen Juckreiz hervorruft [14]. Sie dissimuliert. Zeitweise bedeutet rezidivierend, und ständiger Juckreiz spricht für eine aktive Phase der Erkrankung. In den letzten Lebensjahren machte ihr Arthritis (psoriatica?) zu schaffen. Obwohl sie ihre Psoriasis verbarg und als eher unwesentlich herunterspielte, hatte sie sich offensiv mit ihr auseinander gesetzt.

\section{Das Fotomodell}

Ihr Leben mit der Schuppenflechte schildert Karin Holstein in „Cover Girl Uncovered - A Woman's Journey“ [15]. Schon im Alter von sechs Jahren war das Kind wohlhabender Eltern im Westdeutschland der Nachkriegszeit an Psoriasis erkrankt. Seitdem war das Verbergen befallener Haut eine immer wiederkehrende, qualvolle und perfektionierte Übung im erfolgreichen wie glamourösen Leben des prominenten Fotomodells. Die Behandlung dieser Schuppenflechte kann zunächst mit „Meer und Sonne“ beschrieben werden. Darüber hinaus blieben ihr unangenehme Erinnerungen an eine misslungene Teerbehandlung in der Kindheit. Als Schülerin in Paris überzeugte sie ein befreundeter plastischer Chirurg davon, dass sie die Schuppenflechte mit den Lieblingsspeisen Käsekuchen und Schokolade nie loswerden würde. Stattdessen solle sie Früchte, Gemüse, Körner, Fisch, wenig rotes Fleisch und keine Süßigkeiten essen. Der Wechsel im Speiseplan und viel Bewegung wirkten wunderbar. Begriffe wie „Therapie“ oder „Dermatologe“ kommen im Buch der Enkelin eines Arztes nicht vor.

Es blieb die Furcht vor dem Entblößen des sorgsam Verdeckten. Erst das gefragte Fotomodell erlebte die Befreiung von dieser Furcht. Das geschah unerwartet. Sie war dem Meer und der Sonne mehr als drei Monate fern geblieben und ihre Hände stark von Psoriasis gezeichnet. In dieser Situation hatte ein besonders reputierter Juwelier ein Werbephoto gewünscht, auf dem das Fotomodell mit einem Ring und passenden Ohrringen nebst Kette zu sehen sein sollte. Verzweifelt lehnte sie ab. Sehen Sie sich meine Hände an! Den Juwelier beeindruckte das nicht. Ist das alles? Kein Problem. Wir lieben Ihr wunderbares Gesicht. Wen interessieren schon Ihre Hände! Wir werden ein Hand-Modell heranholen. Mit diesen wenigen Sätzen war jahrelanger Kummer hinweg gefegt. All' die Beleidigungen und die als junges Mädchen erduldeten Hänseleien verschwanden in diesem Moment.

Es bleibt eine Frage. Wurden Dithranol, Jadassohn'sche Kopfsalbe, Kortikosteroide, Etretin, UVB und PUVA nicht erwähnt, weil alle diese komplett versagt hatten? Oder war die intelligente, zielstrebige, energische und wohlhabende Frau jahrzehntelang ausschließlich von Ärzten behandelt worden, deren Spezialkenntnisse Psoriasis nicht betrafen? Prominente scheinen gelegentlich prädestiniert zu sein, von wohlmeinenden, befreundeten oder verwandten, leider aber auch inkompetenten Ärzten abgeschirmt zu werden.

\section{Krankheitsbürrde und Lebensqualität}

Erfreulicherweise nimmt das öffentliche Interesse an der Last, die dem Betroffenen und der Gesellschaft von der Psoriasis aufgebürdet wird, zu. In letzter Zeit wurden mehrere Analysen zum Thema Krankheitslast und Lebensqualität zugänglich. Bei deren Bewertung sollte man die Interessenlage und den Blickwinkel der Untersucher bzw. deren Auftraggeber nicht außer Acht lassen. So interessieren

- die Betroffenen vor allem die krankheitsbedingten Belastungen in Beruf und Privatleben, die sie selbst auch am besten beurteilen können. So kommentiert PSOaktuell - der Ratgeber bei Schuppenflechte - auf Seite 9 in Heft 2/2004 die Aussetzung der Haftstrafe für den Psoriasiskranken und einstigen Firmenchef des Ölkonzerns Elf, Loik Le Floch-Progent: Und das kann ehrlichen Leidensgefährten des Wirtschaftskriminellen in sofern Recht sein, als damit auch juristisch anerkannt wird, dass Schuppenflechte ohne Verschulden schon Strafe genug sein kann

- viele Politiker und diejenigen, die über die Verwendung des bei den Krankenkassen abgelieferten Geldes entscheiden, insbesondere Einsparungsmöglichkeiten

- die Hersteller und Vertreiber von Medikamenten insbesondere die mit maximalem Gewinn medikamentös zu behandelnden Fälle. Und so weiter und so fort.

Hier sollen vor allem Strategie und Taktik des Lebens mit der Krankheit besprochen werden. Sollte man die offensive Auseinandersetzung suchen und als bekennender Psoriatiker auftreten? Oder ist es besser, die kranken Stellen zu verbergen und alle Gedanken an die Krankheit zu verdrängen? Denn auch Gedanken können kränken. Psoriasis ist vielgesichtig [16]. Ihr Spektrum reicht vom nicht diagnostizierten Plaque am Ellenbogen bis zur Erythrodermie mit Arthritis psoriatica. Einige der hier genannten Prominenten litten so stark, dass sie das der Öffentlichkeit mitteilten.

Subjektiv wird auch die schwer verlaufende Psoriasis unterschiedlich bewertet, von davon geht die Welt nicht unter [14] bis zu Inferno [17]. Nicht der objektive Befund ist ausschlaggebend sondern die Krankheitsbürde.

Es fehlt nicht an Versuchen zur Etablierung verlässlicher Maßstäbe, um die Gesamtbelastung durch Psoriasis in Zahlen umsetzen zu können. Das versprechen Verfahren, die eine Gesamtschau über klinischen Schweregrad, Lebensqualität und Dysstress ermöglichen sollen. Die nach wie vor auffälligen Unterschiede zwischen den Ergebnissen der Bestimmung klinischer Schweregrade und denen der überwiegend psychologisch-soziologisch orientierten Methoden wurden als Hinweis auf die Notwendigkeit einer noch umfassenderen Bewertung der Psoriasis interpretiert [18]. Auch das sinnvolle Wichten der Faktoren dürfte eine wahre Kunst sein.

Erhebliche Unterschiede zwischen der messbaren Ausprägung der Erkrankung und der Krankheitsbürde können bereits anhand statistischer Daten definiert werden. Von den 4,5 Millionen Psoriasiskranken in den USA sind mehr als 1 Million mit ihrer gegenwärtigen Behandlung zutiefst unzufrieden; doch nur bei $5 \%$ von letzteren war die Haut großflächig (mehr als drei Handteller) be- 
fallen [19]. Die 3753 befragten, meist leicht bis mittelschwer erkrankten Mitglieder des Deutschen Psoriasisbundes empfanden die Einschränkung ihrer Lebensqualität zu 25\% als geringgradig, zu $60 \%$ als problematisch und zu $15 \%$ als stark [20].

Die besprochenen Biographien Prominenter zeigen, dass diese soweit bekannt - mit ihrer Schuppenflechte um so besser zurecht kamen je früher und intensiver sie sich mit ihr auseinander setzten. Andere verbargen die Schuppenflechte aus Scham oder Karrieregründen und litten deshalb - soweit bekannt - unnötig und übermäßig. Es ist schon erstaunlich, wie wenig auch manche ausgesprochen intelligenten, zielstrebigen und wohlhabenden Personen über ihre Psoriasis wissen.

Dagegen kennt und vermeidet der informierte Psoriasiskranke auslösende Faktoren und weiß um den chronisch-rezidivierenden Verlauf. Er kennt auch zumindest diejenigen therapeutischen Maßnahmen, die ihm besonders gut oder schlecht bekommen. Er hat bessere Chancen, einen spezialisierten Therapeuten zu finden, wie auch therapeutische Handlungen sachkundig vorzunehmen. Therapie-Erfolge geben Lebenskraft. Auch vermeidet der informierte Kranke Enttäuschung infolge unerfüllbarer Erwartungen. Die zitierte [17], tiefe Unzufriedenheit von Kranken mit nur kleinflächigem Befall der Haut reflektiert ein Missverhältnis von Erwartung und Möglichkeit.

Summa summarum sprechen die hier angeführten Biographien dafür, den mühsamen Weg zum informierten Betroffenen um so früher und konsequenter einzuschlagen, je stärker die Krankheitsbürde drückt. Der informierte Psoriatiker kennt seine Krankheit, kann mit ihr und ihrer Behandlung gut umgehen und kämpft erfolgreich um seinen beruflichen und sozialen Status.
Literatur

${ }^{1}$ Willan R. Descriptio and Treatment of Cutaneous Diseases. London: $1798-1807$

${ }^{2}$ Die Bibel. Altes und Neues Testament, Einheitsübersetzung. Der Aussatz an Menschen. Levitikus 13, 42 - 46. Herder-Verlag, Freiburg, Basel, Wien 1980

${ }^{3}$ Die Bibel. Altes und Neues Testament, Einheitsübersetzung. Die Heilung des Aramäres Naaman. 2 Könige 5,1-14. Herder-Verlag, Freiburg, Basel, Wien 1980

${ }^{4}$ Bechstein L. Von dem armen Eli. Der Sagenschatz und die Sagenkreise des Thüringerlandes. Kesselring, Hildburghausen: 1835-1838

${ }^{5}$ Updike J. Wie man Amerika gleichzeitig liebt und verlässt. Verlag Volk und Welt, Berlin 1989

${ }^{6}$ Nabokov V. Ada or Ardor: a family chronicle Penguin Books, London 1971

${ }^{7}$ Meulenberg F. The hidden delight of psorisasis. Brit Med J 1997; 315 : $1709-1711$

8 Vaughan W, Weston H. Jacques-Louis David's Marat. Cambridge Cambridge University Press, 2000

${ }^{9}$ Dotz W. Jean Paul Marat. His life, cutaneous disease, death, and depiction of Jacques Louis David. Am J Dermatopathol 1979; 1: 247-256

10 Beise A, Breuer I. Erläuterungen und Dokumente. Die Verfolgung und Ermordung Jean Paul Marats dargestellt durch die Schauspielgruppe des Hospizes zu Charenton unter Anleitung des Herrn de Sade. Stuttgart Philip Reclam jun, 1995

${ }^{11}$ Bos WH, Farber EM. Joseph Stalin's psoriasis: its treatment and the consequences. Cutis 1997; 59: 197 - 199

${ }^{12}$ Jary M. Ich weiß, es wird einmal ein Wunder gescheh̀n. Die große Liebe der Zarah Leander. Berlin Edition q, 1993

${ }^{13}$ Kleine-Natrop H-E. Psoriasisbehandlung: Ultraviolett, A bis Leander, Z. Dermatol Monatsschr 1976; 162: 633-634

${ }^{14}$ Leander Z. Es war so wunderbar. Mein Leben. Hamburg Hoffmann und Campe, 1973

${ }^{15}$ Holstein K. Cover Girl Uncovered - A Woman's Journey. Winepress Publishing, Washington 2001

${ }^{16}$ Meffert H. Schuppenflechte. Ursachen, Erscheinungen, Behandlung, Bewältigung. Frankfurt am Main und Berlin Ullstein Verlag, 1994

17 Strindberg JA. Lebensgeschichte. Inferno - Legenden. München und Leipzig Georg Müller Verlag, 1920

${ }^{18}$ Sampogna F, Sera F, Abeni D. Measures of clinical severity, quality of life, and psychological distress in patients with psoriasis: a cluster analysis. J invest Dermatol 2004; 122: $602-607$

${ }^{19}$ Stern RS, Nijsten T, Feldman SR, Margolis DJ, Rolstad T. Psoriasis is common, carries a substantial burden even when not extensive, and is associated with widespread treatment dissatisfaction. J Invest Dermatol Symp Proc 2004; 9: 136-139

${ }^{20}$ Redaktionelle Mitteilung. Lebensqualität bei Psoriasis. Aktuelle Dermatologie. 2004; 30: 189-190 